\title{
Phase-controlled spin and charge currents in a superconductor-ferromagnet hybrid
}

\author{
Ali Rezaei $\odot,{ }^{1}$ Robert Hussein, ${ }^{1}$ Akashdeep Kamra $\odot,{ }^{2}$ and Wolfgang Belzig $\odot^{1}$ \\ ${ }^{1}$ Department of Physics, University of Konstanz, 78457 Konstanz, Germany \\ ${ }^{2}$ Center for Quantum Spintronics, Department of Physics, Norwegian University of Science and Technology, 7491 Trondheim, Norway
}

(Received 30 September 2019; revised 12 June 2020; accepted 7 August 2020; published 31 August 2020)

\begin{abstract}
We investigate spin-dependent quasiparticle and Cooper-pair transport through a central node interfaced with two superconductors and two ferromagnets. We demonstrate that voltage biasing of the ferromagnetic contacts induces superconducting triplet correlations on the node and reverses the supercurrent flowing between the two superconducting contacts. We further predict that such triplet correlations can mediate a tunable spin current flow into the ferromagnetic contacts. Our key finding is that noncollinearity in combination with spin-mixing results in equal-spin-triplet correlations on the node and leads to a net charge current between the unbiased two magnets. Our proposed device thus enables the generation, control, and detection of the typically elusive equal-spin-triplet Cooper pairs.
\end{abstract}

DOI: 10.1103/PhysRevResearch.2.033336

\section{INTRODUCTION}

Cooper pairs from a superconductor $(\mathrm{S})$ placed in the vicinity of a ferromagnet $(\mathrm{F})$ may diffuse into the latter, thus modifying their electronic properties [1-5]. The engineering of this proximity effect in hybrid structures generated over the past few years considerable interest in thermoelectricity [6-16], spin caloric transport $[17,18]$, and topological superconductivity [19-24]. Somewhat unexpected is that not only can ferromagnets [25-28] and antiferromagnetic insulators [29] cause spin imbalances into superconductors, but also normal metals [30] exploiting a superconductor itself may serve as a spin filter [31].

By sandwiching a normal metal or ferromagnet between two superconductors, one can realize a Josephson junction featuring a current of Cooper pairs between them, which is characterized by the junctions' free energy [32-34]. Its global minimum determines the ground state of the system. While a ground state at zero phase difference indicates a Josephson current mediated by singlet Cooper pairs, a shifted ground state about $\pi$, occurring in magnetic Josephson junctions [35-44], signals triplet superconductivity [45-48] manifesting in a reversed current phase relation (CPR) [49-51]. Such magnetic Josephson junctions are interesting for quantum computation [52-54] and cryogenic memories [55,56].

Recently, the generation of equal-spin-triplet pairs has been demonstrated in $\mathrm{S} / \mathrm{F}$ structures that contain noncollinear magnetizations [57], as originally predicted by Kadigrobov [58] and Bergeret et al. [59,60]. Mixed-spin-triplet pairs with a zero-spin projection on the $z$ axis, however, already arise for

Published by the American Physical Society under the terms of the Creative Commons Attribution 4.0 International license. Further distribution of this work must maintain attribution to the author(s) and the published article's title, journal citation, and DOI. a homogeneous magnetization. It is the immunity of equalspin-triplet correlations against internal magnetic fields which causes a long penetration length into ferromagnets compared to the ones of singlet and mixed-triplet correlations [61-63]. This property of equal-spin-triplet pairs makes them particular attractive for low-power spintronics [64-66].

In this work, we study spin and charge transport in a four-terminal $\mathrm{S} / \mathrm{F}$ system consisting of two $s$-wave superconducting ( $\left.n=S_{1}, S_{2}\right)$ and two ferromagnetic $\left(n=F_{1}, F_{2}\right)$ terminals with a common contact region [Fig. 1(a)]. Our key finding is that a voltage applied to the two ferromagnetic leads in combination with noncollinear magnetizations can induce triplet superconducting correlations in the system and therewith transitions in the CPR. We show that the mechanism sketched in Fig. 1(b) allows the relative magnetization angle $\theta$ to control the spin currents in the ferromagnetic and superconducting contacts for voltages above the superconducting gap. As shown in the Fig. 1(b), left image, the interaction between the quasiparticle spin chemical potential and the condensate induces triplet correlations which are being spin split and distributed within the superconducting leads, resulting in the net spin supercurrent. Above the superconducting gap, the left and right magnetic leads inject up- and down-spin electrons into the node, respectively [see the right image of Fig. 1(b)]. This implies that there is (an up-)spin flow from the left to the right magnet. Note that $I_{F}^{z}$ is a purely-quasiparticle-mediated spin transport and does not depend on the superconducting phase difference. Finally, we predict that asymmetric spin-mixing conductances (e.g., $G_{1}^{\phi}=0$ and $G_{2}^{\phi}>0$ ) in a noncollinear magnetization configuration leads a finite net charge current between the ferromagnetic contacts. As illustrated in Fig. 1(c), the origin is the generation of equal-spin-triplet correlations in the central node. As depicted in the right image, introducing the finite $G_{2}^{\phi}$ results in an imbalance between the injected Andreev currents to the left and right magnets, which in return gives rise to the current. Altogether, our proposal allows for the experimental detection and exploitation of spin-polarized 

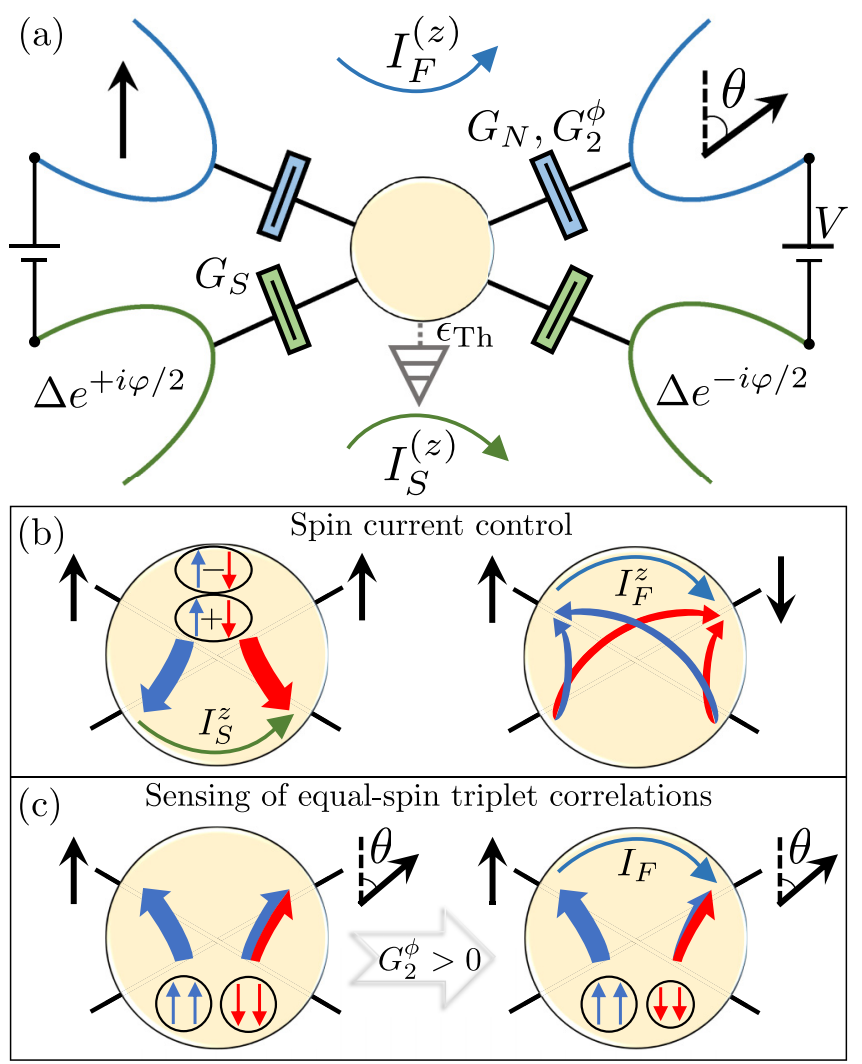

FIG. 1. (a) Four-terminal circuit. Two superconductors with phases $\pm \varphi / 2$ and gap $\Delta$ (green) and two ferromagnets with relative magnetization angle $\theta$ (blue) are connected to a central node (yellow). (b) The left (right) sketch of the central node illustrates a net spin current injection into the superconductors (ferromagnets) due to (anti)parallel magnetization of the ferromagnetic leads for $\epsilon_{\mathrm{Th}} \ll \Delta$ and $V \gtrsim \Delta / e$ in the absence of spin-mixing conductances. Singlet (mixed-triplet) correlations are indicated by encircled arrows containing a minus (plus) sign. (c) Finite spin mixing $G_{2}^{\phi}>0$ allows the generation and detection of equal-spin-triplet correlations (encircled arrows of the same color) by measuring a net charge current between the ferromagnetic leads.

currents by nonequilibrium quasiparticles. In comparison with previous literature $[38,43,46]$ investigating spin-triplet supercurrents via the Josephson effect in $\mathrm{S} / \mathrm{F} / \mathrm{S}$ structures, our proposed device allows an easy voltage control of the spin-triplet correlations and supercurrents. Furthermore, its multiterminal topology allows the currents to be channeled in various ways, making it a potential building block for future superconducting spintronics technologies $[64,65]$.

\section{METHOD}

We study diffusive transport, within a semiclassical [67-71] circuit theory [72,73]. In this framework, hybrid structures are discretized as a network of nodes, terminals, and connectors. Here we map our system to a layout consisting of a central node which is interfaced with two superconducting and two ferromagnetic terminals via corresponding connectors [see Fig. 1(a)]. An additional leakage terminal can account for losses of superconducting correlations. The
Green's function $\breve{\mathcal{G}}_{c}$ of the node, which is an $8 \times 8$ matrix in Keldysh-Nambu spin space, is here the central quantity characterizing the transport properties. The conservation of the total matrix current at this node, $0_{8}=\sum_{n} \check{\mathcal{I}}_{n}$, together with the normalization condition $\breve{\mathcal{G}}_{c}^{2}=\mathbb{1}_{8}$ determines $\breve{\mathcal{G}}_{c}$ and therewith the individual matrix currents $\check{\mathcal{I}}_{n} \equiv\left[\check{\mathcal{M}}_{n}, \check{\mathcal{G}}_{c}\right]$ between terminal $n \in\{S 1, S 2, F 1, F 2$, leak $\}$ and the central node. ${ }^{1}$

The superconducting contacts are characterized by the BCS bulk Green's functions $\left(2 / G_{S}\right) \check{\mathcal{M}}_{S \alpha}=\breve{\mathcal{G}}_{S \alpha}$, with $G_{S}$ the conductance of the corresponding connector to the central node and $\alpha=1,2$. Its retarded/advanced component reads, in the spinor basis $\left\{\Psi_{\uparrow}^{\dagger}, \Psi_{\downarrow}^{\dagger}, \Psi_{\downarrow},-\Psi_{\uparrow}\right\}[6,7]$,

$$
\hat{\mathcal{G}}_{S \alpha}^{R, A}=\frac{ \pm \operatorname{sgn}(\epsilon)}{\sqrt{(\epsilon \pm i \Gamma)^{2}-\left|\Delta_{\alpha}\right|^{2}}}\left(\begin{array}{cc} 
\pm i \Gamma+\epsilon & \Delta_{\alpha} \\
-\Delta_{\alpha}^{*} & \mp i \Gamma-\epsilon
\end{array}\right) \otimes \mathbb{1}_{2},
$$

where $\Delta_{1,2} \equiv \Delta \exp [ \pm i \varphi / 2]$ denotes the superconducting gap with phase difference $\varphi$ across the junction. A small imaginary component in the denominator of Eq. (1) (here $\Gamma=10^{-3} \Delta$ ) accounts for a finite lifetime $\hbar / \Gamma$ of the quasiparticles, with energy $\epsilon$, smearing out the superconducting gap [74]. The ferromagnetic contacts are governed by $\check{\mathcal{M}}_{F \alpha}=(1 / 2)\left[G_{N}\left(\mathbb{1}_{8}+\right.\right.$ $\left.\left.P \breve{\kappa}_{\alpha}\right) \breve{\mathcal{G}}_{F}-i G_{\alpha}^{\phi} \breve{\kappa}_{\alpha}\right]$, with $G_{N}\left(G_{\alpha}^{\phi}\right)$ the normal (spin-mixing) conductances of the corresponding connectors. In addition, $P$ denotes the contact spin polarization and $\check{\kappa}_{\alpha}=\mathbb{1}_{2} \otimes \sigma_{z} \otimes$ $\left(\boldsymbol{m}_{\alpha} \cdot \boldsymbol{\sigma}\right)$ is the spin matrix which is diagonal in Keldysh space. In the last expression, $\sigma=\left\{\sigma_{x}, \sigma_{y}, \sigma_{z}\right\}$ labels the vector of Pauli matrices and $\boldsymbol{m}_{\alpha}$ is the magnetization vector corresponding to the ferromagnet $F \alpha$. Here we set $\boldsymbol{m}_{1}=(0,0,1)$ in the $z$ direction and consider $\boldsymbol{m}_{2}=(\sin \theta, 0, \cos \theta)$ tilted by an arbitrary angle $\theta$. We further consider fully polarized ferromagnetic contacts, i.e., $P=1$. The retarded/advanced component of the ferromagnetic Green's function is given by $\hat{\mathcal{G}}_{F}^{R, A}= \pm \sigma_{z} \otimes \mathbb{1}_{2}$. Finally, the leakage terminal is described by $\check{\mathcal{M}}_{\text {Leak }}=-i G_{S}\left(\epsilon / \epsilon_{\mathrm{Th}}\right) \mathbb{1}_{2} \otimes \sigma_{z} \otimes \mathbb{1}_{2}$, with $\epsilon_{\mathrm{Th}}$ the Thouless energy. The Keldysh component of the $\mathrm{S}$ and $\mathrm{F}$ Green's functions follows from $\hat{\mathcal{G}}_{n}^{K}=\hat{\mathcal{G}}_{n}^{R} \hat{h}_{n}-\hat{h}_{n} \hat{\mathcal{G}}_{n}^{A}$, with the distribution function $\hat{h}_{n}=\operatorname{diag}\left(\tanh \left[\left(\epsilon-e V_{n}\right) / 2 k_{B} T\right], \tanh [(\epsilon+\right.$ $\left.\left.\left.e V_{n}\right) / 2 k_{B} T\right]\right) \otimes \mathbb{1}_{2}$. Hereafter, we assume all contacts at zero temperature $k_{B} T=0$, the superconductors at zero (reference) voltage $V_{S 1}=V_{S 2}=0$, equally biased ferromagnetic contacts $V \equiv V_{F 1}=V_{F 2}$, and equal conductances $G_{S}=G_{N}$. The cases of asymmetric device configurations and partially polarized ferromagnets are discussed in Appendixes A and B, respectively.

The Keldysh component of the matrix currents $\check{\mathcal{I}}_{n}$ leads to the charge currents

$$
I_{n}=\frac{1}{8 e} \int_{-\infty}^{\infty} d \epsilon \operatorname{tr}\left[\left(\sigma_{z} \otimes \mathbb{1}_{2}\right) \hat{I}_{n}^{K}(\epsilon)\right]
$$

and the $z$-polarized spin currents

$$
I_{n}^{z}=\frac{\hbar}{16 e^{2}} \int_{-\infty}^{\infty} d \epsilon \operatorname{tr}\left[\left(\mathbb{1}_{2} \otimes \sigma_{z}\right) \hat{I}_{n}^{K}(\epsilon)\right]
$$

\footnotetext{
${ }^{1}$ The symbol $0_{n}\left(\mathbb{1}_{n}\right)$ labels the zero (identity) matrix in $n \times n$ dimensions and the haček indicates the Keldysh $\otimes \mathrm{Nambu} \otimes$ spin space of dimension $8 \times 8$.
} 

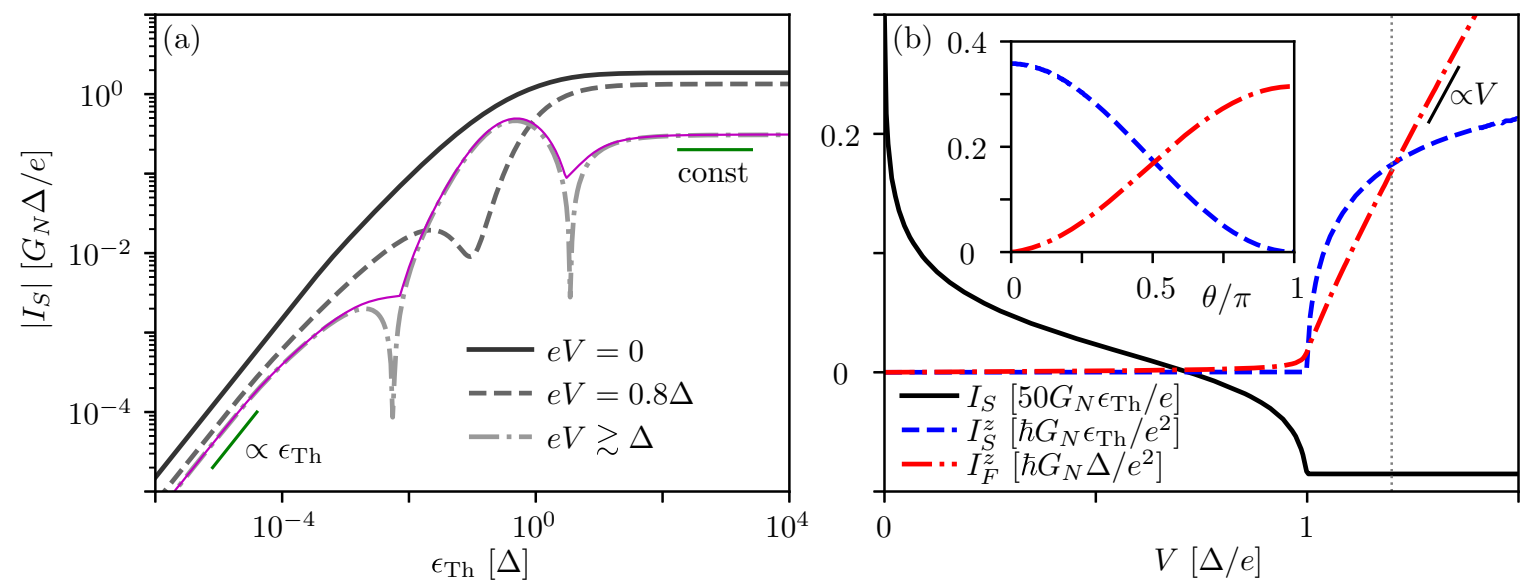

FIG. 2. (a) Modulus of the net supercurrent $I_{S}$ as a function of the Thouless energy $\epsilon_{\mathrm{Th}}$ for different voltages $V$. The divergencies occurring for large voltages $V \gtrsim \Delta / e$ indicate $0-\pi$ transitions. The parameters are $\theta=0, \varphi=\pi / 2$, and $G_{1}^{\phi}=G_{2}^{\phi}=0$. The thin purple line indicates the corresponding critical current $I_{C}=\max _{\varphi} I_{S}$ for $V \gtrsim \Delta / e$. (b) Net supercurrent $I_{S}$ and net spin currents $I_{S}^{z}$ and $I_{F}^{z}$ between the superconductors/ferromagnets for $\theta=\varphi=\pi / 2$ and $\epsilon_{\mathrm{Th}} \ll \Delta$. The inset shows the net spin currents as a function of $\theta$ for $V=1.2 \Delta / e$, as indicated by the horizontal dotted line in (b).

[73,75-77]. In particular, we will analyze the charge $I_{X}=$ $I_{X 1}-I_{X 2}$ and spin net current $I_{X}^{z}=I_{X 1}^{z}-I_{X 2}^{z}$ between the superconductors/ferromagnets $(X=S, F)$. The matrix elements $f_{s s^{\prime}}=\left\langle\Psi_{s}\left|\hat{\mathcal{G}}_{c}^{K}\right| \Psi_{s^{\prime}}\right\rangle$, with $s, s^{\prime}=\uparrow, \downarrow$, contain the spectral information about spin-pair correlations in nonequilibrium. Here we consider the integrated quantities over positive energies $\epsilon>0$ (triplet correlations are odd in $\epsilon$ ) to quantify singlet $F_{S}=\int d \epsilon\left(f_{\uparrow \downarrow}-f_{\downarrow \uparrow}\right) / \sqrt{2}$, mixed-spin triplet $F_{T 0}=\int d \epsilon\left(f_{\uparrow \downarrow}+f_{\downarrow \uparrow}\right) / \sqrt{2}$, and equal-spin-triplet correlations $F_{T s}=\int d \epsilon f_{s s}$.

\section{SCALING}

Before analyzing the interplay between Cooper-pair and quasiparticle transport, let us recall that in equilibrium only a Josephson current $I_{S}^{\mathrm{eq}}=I_{C} \sin \varphi$ may flow between both $s$-wave superconductors, which has a purely sinusoidal CPR; all other currents require quasiparticle excitations. Depending on the effective size $L$ of the central node, the Josephson current scales in the diffusive regime for $\epsilon_{\mathrm{Th}} \ll \Delta$ (large island) with the Thouless energy $\epsilon_{\mathrm{Th}} \equiv \hbar D / L^{2}$, where $D$ is the diffusion constant [see the black solid line in Fig. 2(a)]. For $\epsilon_{\mathrm{Th}} \gg \Delta$ (small island), however, it is characterized by the superconducting gap $\Delta$ [34]. While the superconducting condensate is in equilibrium entirely formed by spin-singlet Cooper pairs, finite voltages may cause triplet correlations in the system. They can lead, for voltages below the gap [dashed line in Fig. 2(a)], to a reduction of the Josephson current; here we consider parallel collinear magnetization. For voltages above the gap and intermediate values of the Thouless energy $\epsilon_{\mathrm{Th}}$, such triplet correlations can even induce current reversals in the Josephson current $I_{S}$. In Fig. 2(a), showing the modulus of $I_{S}$ on a double logarithmic scale, these zero crossings (at which the logarithm diverges) result in the two sharp dips of the dash-dotted curve. Also the corresponding critical current $I_{C}=\max _{\varphi} I_{S}$ [purple line in Fig. 2(a)] indicates with the kinks the presence of triplet correlations.

For Thouless energies much smaller (larger) than the superconducting gap, the corresponding net supercurrent stays always positive and is dominated by spin-singlet correlations. We show in the following that applied voltages in combination with nonparallel magnetization $\theta \neq 0$ can induce triplet correlations and transitions in the CPR also in the regimes $\epsilon_{\mathrm{Th}} \ll \Delta$ and $\epsilon_{\mathrm{Th}} \gg \Delta$.

\section{PHASE TRANSITIONS AND SPIN CURRENT CONTROL}

First, let us consider the large-island regime $\epsilon_{\mathrm{Th}} \ll \Delta$, where the Cooper-pair transport is characterized by the Thouless energy, i.e., $I_{S}, I_{S}^{z} \propto \epsilon_{\mathrm{Th}}$. While the net current between both superconductors follows in equilibrium the usual sinusoidal CPR, $I_{S} \propto \sin \varphi$, nonequilibrium in combination with noncollinear magnetization, $0<\theta<\pi$, can induce $0-\pi$ transitions for voltages $V>0$ (and $P \gtrsim 0.6$; cf. Appendix B) below the gap $\Delta$, due to mixed-spin-triplet correlations [black solid line in Fig. 2(b)]. Above the gap, quasiparticle transport sets in and $I_{S}$ saturates. In this regime, a finite net spin current $I_{F}^{z} \propto V$ (red dash-dotted line) emerges between the ferromagnetic contacts for a nonzero magnetization angle, irrespective of $\varphi$. For the chosen symmetric configuration, however, no corresponding net charge current $I_{F}$ flows.

A special feature of our setup is the occurrence of a finite net spin current $I_{S}^{z}$ between both superconductors (blue dashed line) for voltages $V \gtrsim \Delta / e$. This effect is maximal for parallel magnetization $\theta=0$, as can be seen in the inset, for which only mixed-triplet and singlet correlations are present. It vanishes for antiparallel orientation $\theta=\pi$, where no triplet correlations arise, and when the Josephson phase $\varphi$ is a multiple of $\pi$. Notice that $I_{S}^{z}$ is antisymmetric in $\varphi$, and $I_{S}^{z}$ and $I_{F}^{z}$ are symmetric in $\theta$. While voltages $V$ above the gap can trigger net spin currents $I_{F}^{z}$ and $I_{S}^{z}$, the magnetization angle $\theta$ can control their ratio (see the inset), making the proposed setup thus attractive for future applications.

\section{SPIN-MIXING-INDUCED CHARGE CURRENT}

Let us now turn to the small-island regime $\epsilon_{\mathrm{Th}} \gg$ $\Delta$, where losses of superconducting coherence become 

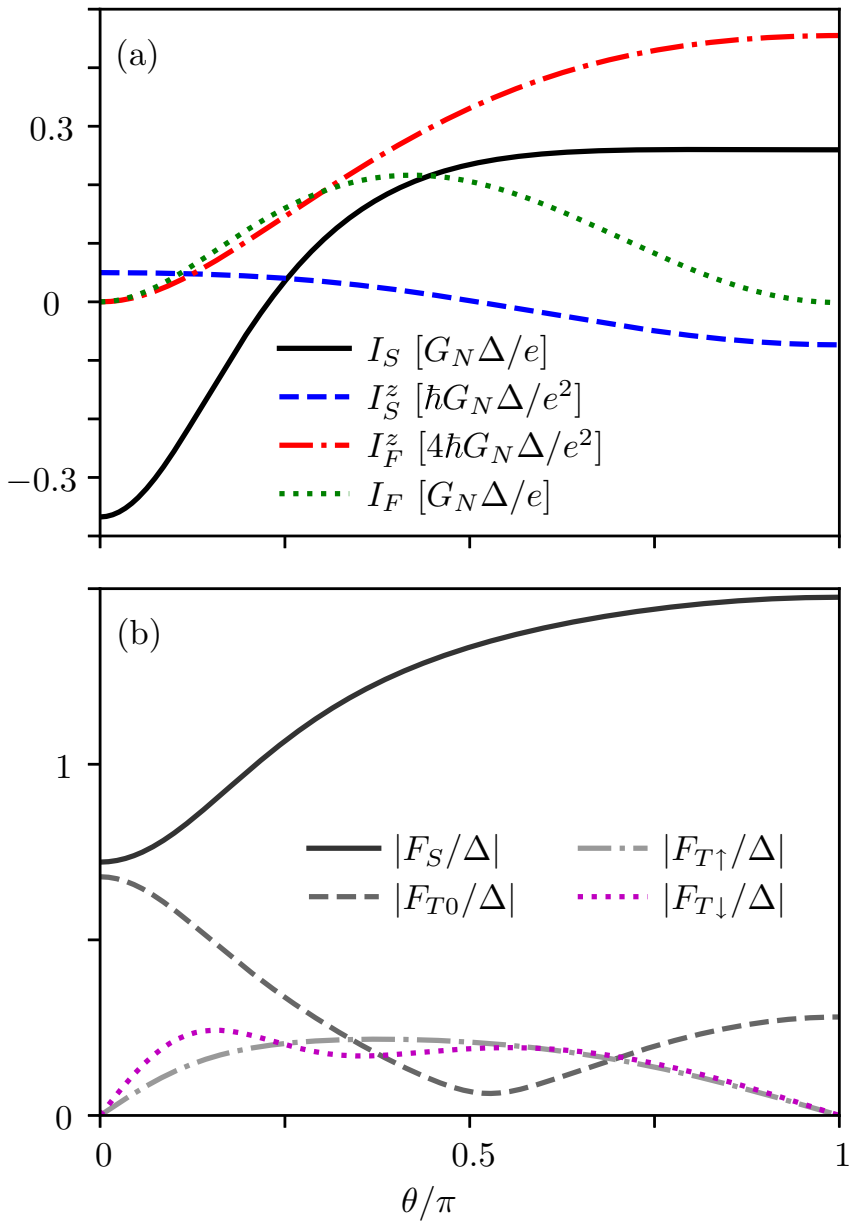

FIG. 3. (a) Net charges $I_{S}$ and $I_{F}$ and net spin currents $I_{S}^{z}$ and $I_{F}^{z}$ as a function of $\theta$ for $\epsilon_{\mathrm{Th}} \gg \Delta, \varphi=\pi / 2, V=2 \Delta / e, G_{1}^{\phi}=0$, and $G_{2}^{\phi}=2 G_{N}$. (b) Modulus of the spin singlet $F_{S}$ and the spin-triplet pairing functions $F_{T 0}, F_{T \uparrow}$, and $F_{T \downarrow}$. Notice that the finite spin-mixing conductance $G_{2}^{\phi}$ induces a net charge current $I_{F}$ [in (a)] following the curve progression of the equal-spin-triplet correlations, thus making them experimentally attainable by standard current measurements [see Fig. 1(c) for $\theta \approx 0.3 \pi$, where $\left|F_{T \downarrow}\right|<\left|F_{T \uparrow}\right|$ ].

irrelevant and the Josephson transport is characterized by the gap energy, i.e., $I_{S}, I_{S}^{z} \propto \Delta$. Here we find that nonantiparallel magnetization $\theta \neq \pi$ can indeed induce triplet correlations for sufficiently large voltages, which results in an asymmetric sinusoidal CPR. However, it cannot induce $0-\pi$ transitions. To cure this circumstance, we consider in the following finite spin mixing for voltages above the gap. Indeed, Fig. 3(a) indicates that the system is for parallel magnetization $\theta=0$, but finite spin mixing $G_{2}^{\phi}>0$ in a $\pi$ phase featuring a negative net supercurrent $I_{S}$ (black solid line). Here we consider the spinmixing conductance $G_{2}^{\phi}$ of the order of the normal one [57]. Roughly at $\theta=\pi / 4$, the CPR undergoes a $\pi-0$ transition. Similar to the large-island regime [inset of Fig. 2(b)], a net spin current $I_{F}^{z}$ may flow between the ferromagnets [red dashdotted line in Fig. 3(a)] which is essentially unaffected by spin-mixing. The net spin current $I_{S}^{z}$ between the superconductors (blue dashed line), on the contrary, is apart from $\theta=0$ modified by spin mixing and features a current reversal about $\theta=\pi / 2$.
Remarkably, the noncollinear magnetization in this system gives rise to equal-spin-triplet correlations $\left|F_{T \uparrow}\right|$ and $\left|F_{T \downarrow}\right|$ [see Fig. 3(b)]. A distinctive feature, however, is that these equal-spin-triplet correlations can induce a finite net charge current $I_{F}$ into the ferromagnets [green dotted line in Fig. 3(a)] for asymmetric spin mixing $G_{1}^{\phi} \neq G_{2}^{\phi}$. This feature is attributed to the creation of an imbalance in the ferromagnetic spin channels [see Fig. 1(c)]. This effect also persists for vanishing Josephson phase $\varphi$. Under a mutual exchange of the spin-mixing conductances $\left(G_{1}^{\phi} \leftrightarrow G_{2}^{\phi}\right)$, the net charge current $I_{F}$ just inverts. However, $I_{F}$ additionally contains an offset for asymmetric device configurations, detailed in Appendix A. An experimentally measurable charge current $I_{F}$ serves also in the large-island regime as a signature of equal-spin-triplet correlations. It features in this regime a similar curve progression, but scales instead with $\epsilon_{\mathrm{Th}}$.

\section{CONCLUSION}

Spin-dependent quasiparticle and Cooper-pair transport have been analyzed in a proximity-coupled multiterminal $\mathrm{S} / \mathrm{F}$ heterostructure in nonequilibrium. We have shown that $0-\pi$ transitions can be induced in the CPR by biasing the ferromagnetic contacts and bearing noncollinear magnetic moments, as long as the loss of superconducting coherence is large, $\epsilon_{\mathrm{Th}} \ll \Delta$. In this limit, voltages exceeding the superconducting gap, $V \gtrsim \Delta / e$, trigger net spin currents into the ferromagnets/superconductors, which can be controlled by the relative magnetization angle $\theta$. The small-island regime, however, requires additionally finite spin mixing to induce a $0-\pi$ transition in the CPR. The proposed heterostructure constitutes an ideal platform for the generation of triplet correlations of different spin projection and as a voltageand phase-controlled switch for spin and electron currents. In particular, it constitutes a minimal setup for generating, controlling, and detecting equal-spin-triplet correlations by current measurements.

\section{ACKNOWLEDGMENTS}

This work was financially supported by the DFG through SFB 767, the Carl-Zeiss-Stiftung, the Alexander von Humboldt Foundation, and the Research Council of Norway through its Centers of Excellence funding scheme, Project No. 262633, "QuSpin.” A.R. and W.B. gratefully acknowledge the hospitality of QuSpin (NTNU) during their visit to Trondheim.

\section{APPENDIX A: ASYMMETRIC DEVICE SETUP}

In this Appendix, we address the effect of an asymmetric setup and discuss in particular asymmetric boundary conductances of the connectors between the central node and the ferromagnetic/superconducting terminals. Unlike the voltages, they cannot be tuned externally and differ from sample to sample, although a low asymmetry can generally be achieved within a single device and fabrication process. We distinguish here the conductances to the ferromagnetic terminals $n=F \alpha$ with $G_{N \alpha}(\alpha=1,2)$ and define the conductances to the superconducting terminals $n=S \alpha$ with $G_{S \alpha}$. Note that 

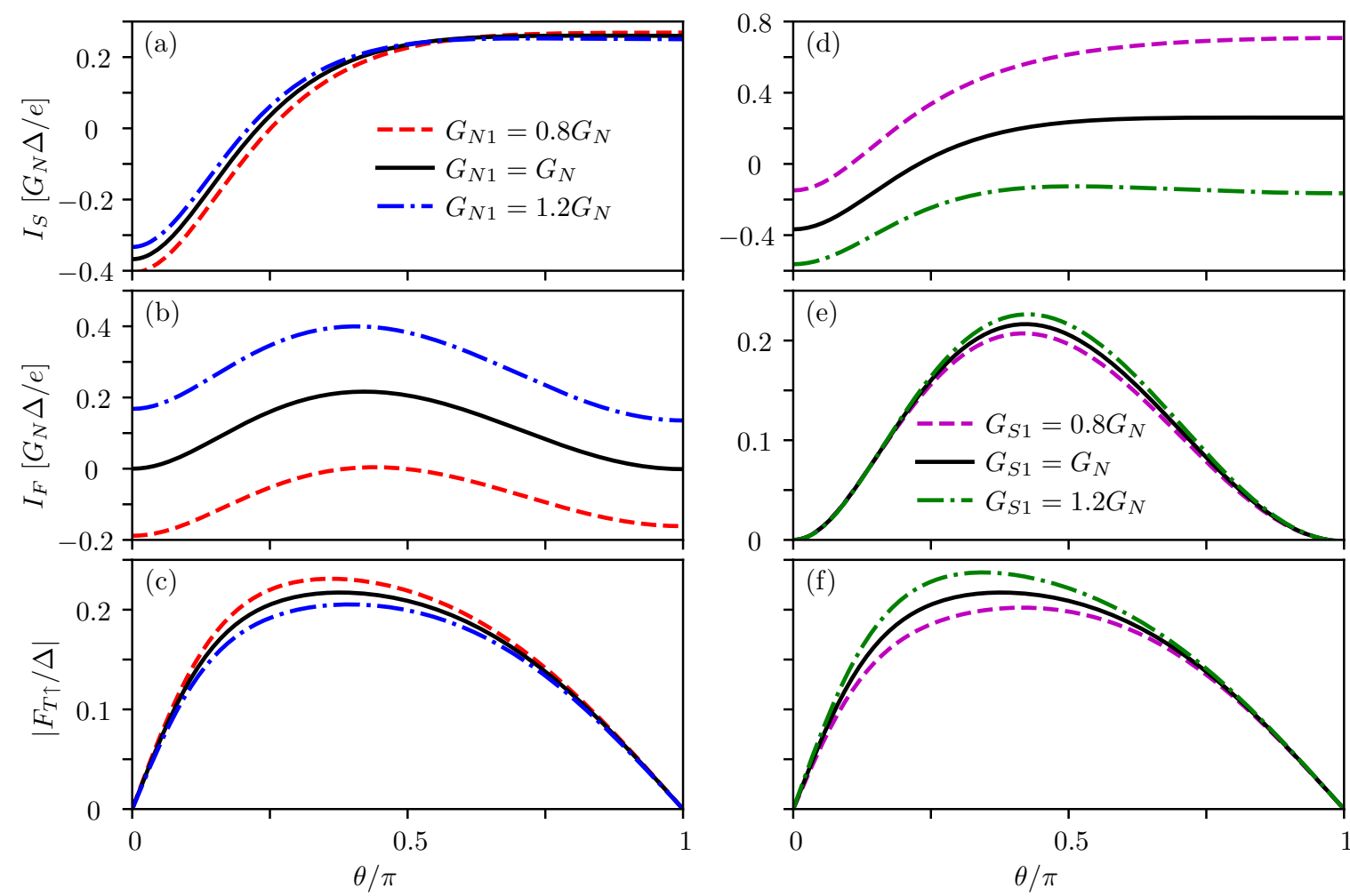

FIG. 4. (a) and (d) Net supercurrent $I_{S}$, (b) and (e) net charge current $I_{F}$, and (c) and (f) equal-spin-triplet correlation $F_{T \uparrow}$ as a function of $\theta$. In (a)-(c) we consider different $G_{N 1}$ but $G_{S 1}=G_{S 2}$. Conversely, we consider in (d)-(f) different $G_{S 1}$ but $G_{N 1}=G_{N 2}$. The parameters are $\epsilon_{\mathrm{Th}} \gg \Delta, \varphi=\pi / 2, V=2 \Delta / e, G_{1}^{\phi}=0, G_{2}^{\phi}=2 G_{N}$, and $G_{S 2}=G_{N}$ with $G_{N} \equiv G_{N 2}$. The black lines indicate the symmetric configuration.

these four conductances have been chosen to be equal in the main text and hence are denoted therein by the same symbol $G_{N}$.

Let us first consider the case of asymmetric conductances of the connectors to the ferromagnets. Figure 4(a) shows that the net supercurrent $I_{S}$ for asymmetric conductances $G_{N 1} \neq$ $G_{N 2}$ (red dashed line and blue dash-dotted line) only slightly deviates from the symmetric case (black solid line). The occurrence of equal-spin-triplet correlations $F_{T \uparrow}$ is comparably robust, as can be seen in Fig. 4(c). On the contrary, the net charge current $I_{F}$ [Fig. 4(b)] exhibits an additional offset current which is independent of the relative magnetization angle $\theta$. Thus, the mere occurrence of a finite net charge current $I_{F}$ does not suffice as an indicator for equal-spin-triplet correlations for an asymmetric setup. In other words, the offset current, which in our normalized units is comparable to the relative asymmetry ( $\sim 20 \%$ in Fig. 4 ), needs to be lower than the current driven by the triplet correlations. This provides experimental guidance for the maximum $G_{N}$ asymmetry allowed in the device. The experimentally realized asymmetry can be rather small with contemporary in situ fabrication methods and may not pose a hurdle in the suggested detection scheme. The offset originates in the asymmetry-induced imbalance between the quasiparticle flows through each ferromagnet which results in a net charge current $I_{F}^{\text {offset }} \approx\left(G_{N 1}-G_{N 2}\right) V / 2$.

Now let us turn to the case of asymmetric conductances of the connectors to the superconductors. Here the net charge current $I_{F}$ [colored lines in Fig. 4(e)] and the equal-spin- triplet correlations $F_{T \uparrow}$ [colored lines in Fig. 4(f)] show only small deviations from the symmetric configuration (black lines). However, the net supercurrent is roughly shifted due to the asymmetry $G_{S 1} \neq G_{S 2}$ with an offset of $I_{S}^{\text {offset }} \approx\left(G_{S 1}-\right.$ $\left.G_{S 2}\right) V$ [see Fig. 4(d)]. Here we have to subtract this offset contribution from $I_{S}$ to obtain the supercurrent from which one can estimate the $0-\pi$ transitions.

\section{APPENDIX B: PARTIALLY-SPIN-POLARIZED FERROMAGNETS}

While we focused in the main text on the ideal case of fully polarized ferromagnets permitting us to more easily separate occurring transport processes, we discuss in this Appendix what changes when rather partially-spin-polarized ones are used. In particular, we consider here typical ferromagnets such as $\mathrm{Ni}, \mathrm{Co}$, and $\mathrm{Fe}$, which have a spin polarization of about $P=0.3-0.5$; higher values may be achieved by using specific compound semiconductors or alloys $[38,78,79]$.

To get an overview of the chief differences with respect to the fully polarized case, we depict in Fig. 5(a) how the charge and spin currents, given in Fig. 3(a), change for a polarization of $P=0.4$. Note that we have also adapted the phase difference (here $\varphi=0.23$ ) in order to minimize the supercurrent (black solid line). However, before elaborating upon it, we note that the charge current $I_{F}$ (green dotted line) and the spin currents $I_{F}^{z}$ (red dash-dotted line) and $I_{S}^{z}$ (blue dashed line) are mainly reduced compared to the 

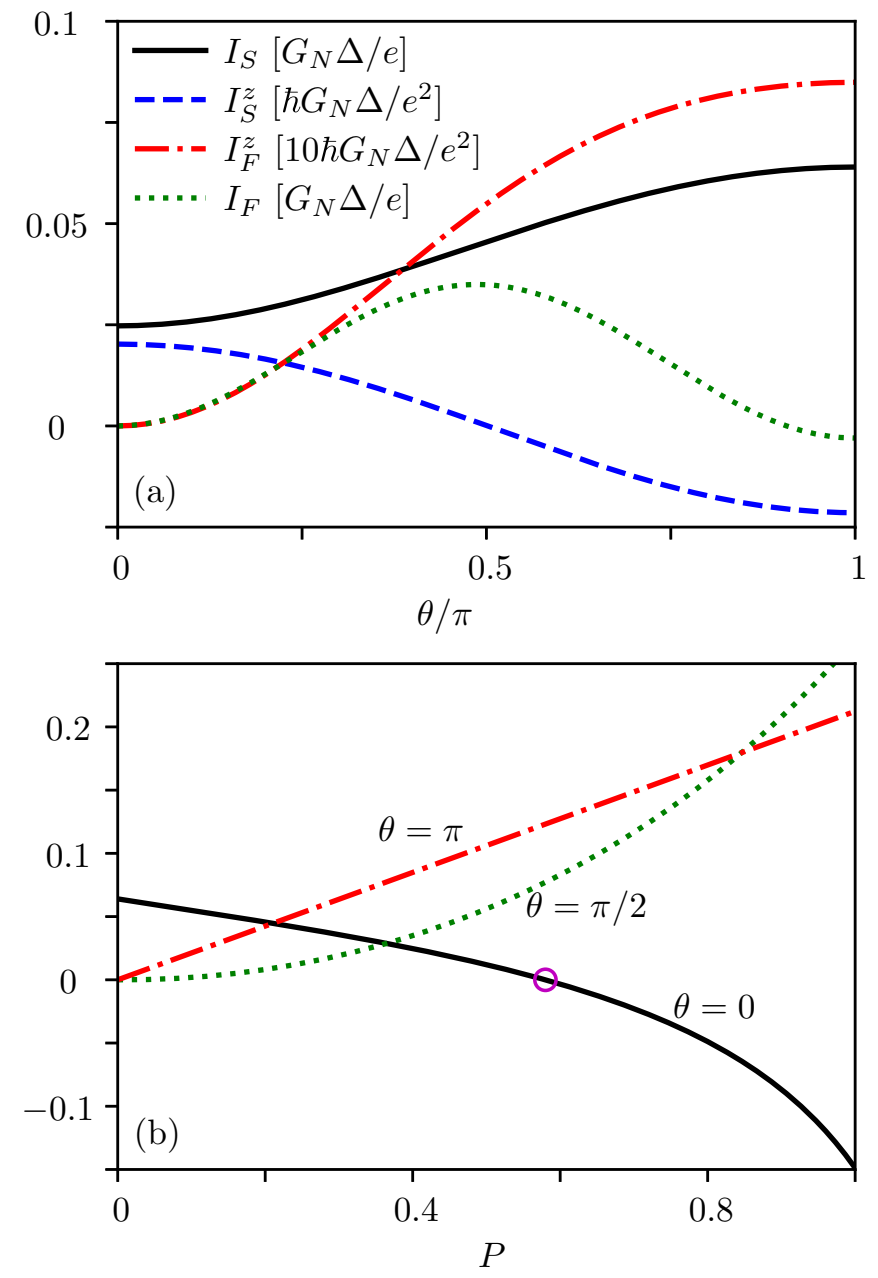

FIG. 5. (a) Net charges $I_{S}$ and $I_{F}$ and net spin currents $I_{S}^{z}$ and $I_{F}^{z}$ as a function of $\theta$ for the spin polarization $P=0.4$. (b) Corresponding $P$ dependence of $I_{S}(\theta=0), I_{F}(\theta=\pi / 2)$, and $I_{F}^{z}(\theta=\pi)$. We have set the other parameters to $\epsilon_{\mathrm{Th}} \gg \Delta, \varphi=0.23, V=2 \Delta / e, G_{1}^{\phi}=0$, and $G_{2}^{\phi}=2 G_{N}$. The magenta circle indicates the minimum value of $P$ for which the supercurrent changes sign, thereby manifesting a $0-\pi$ transition.

fully polarized case. However, there is no global rescaling factor. While the net charge current $I_{F}$ scales roughly

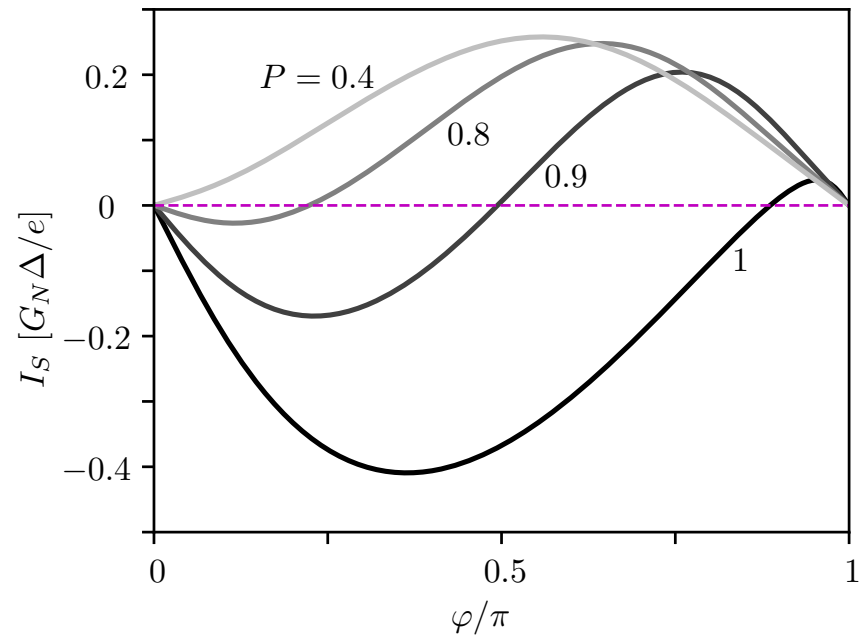

FIG. 6. Current phase relation of $I_{S}$ for different spin polarizations and parallel magnetization $\theta=0$. The other parameters are as in Fig. 5.

quadratically with $P$ [green dotted line in Fig. 5(b)], the net spin current $I_{F}^{z}$ changes rather linearly [red dash-dotted line in Fig. 5(b)].

Now let us turn to the net supercurrent $I_{S}$, which for a spin polarization of $P=0.4$ no longer features a current reversal [see Fig. 5(a)]. This disappearance is just related to the fact that a weaker polarization also implies that the triplet correlations are less pronounced. Figure 5(b) reflects this interplay between the singlet and the triplet correlations in the monotonic rise of $I_{S}$ for a decreasing $P$. It can be seen that the system stays for a polarizations below $P \approx 0.6$ (purple circle) always in a 0-like state. Finally, let us return to the choice of the phase difference used in Fig. 5. To this aim, we depict in Fig. 6 the current-phase relation of the net supercurrent $I_{S}$ for different spin polarizations. We see that the $-\sin \varphi$ behavior for strong polarization $(P=1)$ toward a $\sin \varphi$ behavior for weak polarization $(P=0.4)$ goes along with a shift of the minimum occurring at smaller values of $\varphi$. Moreover, one sees that for an intermediate spin polarization of $P=0.9$ the net supercurrent $I_{S}$ picks up a large $-\sin 2 \varphi$ component.
[1] H. Meissner, Superconductivity of contacts with interposed barriers, Phys. Rev. 117, 672 (1960).

[2] P. G. de Gennes, Boundary effects in superconductors, Rev. Mod. Phys. 36, 225 (1964).

[3] W. L. McMillan, Tunneling model of the superconducting proximity effect, Phys. Rev. 175, 537 (1968).

[4] T. M. Klapwijk, Proximity effect from an Andreev perspective, J. Supercond. 17, 593 (2004).

[5] A. I. Buzdin, Proximity effects in superconductor-ferromagnet heterostructures, Rev. Mod. Phys. 77, 935 (2005).

[6] P. Machon, M. Eschrig, and W. Belzig, Nonlocal Thermoelectric Effects and Nonlocal Onsager Relations in a ThreeTerminal Proximity-Coupled Superconductor-Ferromagnet Device, Phys. Rev. Lett. 110, 047002 (2013).
[7] P. Machon, M. Eschrig, and W. Belzig, Giant thermoelectric effects in a proximity-coupled superconductor-ferromagnet device, New J. Phys. 16, 073002 (2014).

[8] A. Ozaeta, P. Virtanen, F. S. Bergeret, and T. T. Heikkilä, Predicted Very Large Thermoelectric Effect in FerromagnetSuperconductor Junctions in the Presence of a SpinSplitting Magnetic Field, Phys. Rev. Lett. 112, 057001 (2014).

[9] F. Giazotto, P. Solinas, A. Braggio, and F. S. Bergeret, Ferromagnetic-Insulator-Based Superconducting Junctions as Sensitive Electron Thermometers, Phys. Rev. Appl. 4, 044016 (2015).

[10] S. Kolenda, M. J. Wolf, and D. Beckmann, Observation of Thermoelectric Currents in High-Field Superconductor- 
Ferromagnet Tunnel Junctions, Phys. Rev. Lett. 116, 097001 (2016).

[11] S. Kolenda, P. Machon, D. Beckmann, and W. Belzig, Nonlinear thermoelectric effects in high-field superconductor-ferromagnet tunnel junctions, Beilstein J. Nanotechnol. 7, 1579 (2016).

[12] S. Kolenda, C. Sürgers, G. Fischer, and D. Beckmann, Thermoelectric effects in superconductor-ferromagnet tunnel junctions on europium sulfide, Phys. Rev. B 95, 224505 (2017).

[13] A Rezaei, A Kamra, P Machon, and W Belzig, Spin-flip enhanced thermoelectricity in superconductor-ferromagnet bilayers, New J. Phys. 20, 073034 (2018).

[14] R. Sánchez, P. Burset, and A. L. Yeyati, Cooling by Cooper pair splitting, Phys. Rev. B 98, 241414(R) (2018).

[15] R. Hussein, M. Governale, S. Kohler, W. Belzig, F. Giazotto, and A. Braggio, Nonlocal thermoelectricity in a Cooper-pair splitter, Phys. Rev. B 99, 075429 (2019).

[16] N. S. Kirsanov, Z. B. Tan, D. S. Golubev, P. J. Hakonen, and G. B. Lesovik, Heat switch and thermoelectric effects based on Cooper-pair splitting and elastic cotunneling, Phys. Rev. B 99, 115127 (2019).

[17] J. Linder and M. E. Bathen, Spin caloritronics with superconductors: Enhanced thermoelectric effects, generalized Onsager response-matrix, and thermal spin currents, Phys. Rev. B 93, 224509 (2016).

[18] M. E. Bathen and J. Linder, Spin Seebeck effect and thermoelectric phenomena in superconducting hybrids with magnetic textures or spin-orbit coupling, Sci. Rep. 7, 41409 (2017).

[19] W. S. Cole, S. Das Sarma, and T. D. Stanescu, Effects of large induced superconducting gap on semiconductor Majorana nanowires, Phys. Rev. B 92, 174511 (2015).

[20] C.-K. Chiu, W. S. Cole, and S. Das Sarma, Induced spectral gap and pairing correlations from superconducting proximity effect, Phys. Rev. B 94, 125304 (2016).

[21] K. Halterman and M. Alidoust, Induced energy gap in finitesized superconductor/ferromagnet hybrids, Phys. Rev. B 98, 134510 (2018).

[22] H. Ren, F. Pientka, S. Hart, A. T. Pierce, M. Kosowsky, L. Lunczer, R. Schlereth, B. Scharf, E. M. Hankiewicz, L. W. Molenkamp, B. I. Halperin, and A. Yacoby, Topological superconductivity in a phase-controlled Josephson junction, Nature (London) 569, 93 (2019).

[23] G. Blasi, F. Taddei, V. Giovannetti, and A. Braggio, Manipulation of Cooper pair entanglement in hybrid topological Josephson junctions, Phys. Rev. B 99, 064514 (2019).

[24] T. Kiendl, F. von Oppen, and P. W. Brouwer, Proximity-induced gap in nanowires with a thin superconducting shell, Phys. Rev. B 100, 035426 (2019).

[25] M. Johnson, Spin coupled resistance observed in ferromagnetsuperconductor-ferromagnet trilayers, Appl. Phys. Lett. 65, 1460 (1994).

[26] C. H. L. Quay, D. Chevallier, C. Bena, and M. Aprili, Spin imbalance and spin-charge separation in a mesoscopic superconductor, Nat. Phys. 9, 84 (2013).

[27] F. S. Bergeret, M. Silaev, P. Virtanen, and T. T. Heikkilä, Colloquium: Nonequilibrium effects in superconductors with a spin-splitting field, Rev. Mod. Phys. 90, 041001 (2018).

[28] T. T. Heikkilä, M. Silaev, P. Virtanen, and F. S. Bergeret, Thermal, electric and spin transport in superconductor/ ferromagnetic-insulator structures, Prog. Surf. Sci. 94, 100540 (2019).
[29] A. Kamra, A. Rezaei, and W. Belzig, Spin Splitting Induced in a Superconductor by an Antiferromagnetic Insulator, Phys. Rev. Lett. 121, 247702 (2018).

[30] M. J. Wolf, F. Hübler, S. Kolenda, H. v. Löhneysen, and D. Beckmann, Spin injection from a normal metal into a mesoscopic superconductor, Phys. Rev. B 87, 024517 (2013).

[31] F. Hübler, M. J. Wolf, D. Beckmann, and H. v. Löhneysen, Long-Range Spin-Polarized Quasiparticle Transport in Mesoscopic Al Superconductors with a Zeeman Splitting, Phys. Rev. Lett. 109, 207001 (2012).

[32] B. D. Josephson, Supercurrents through barriers, Adv. Phys. 14, 419 (1965).

[33] F. Bloch, Josephson effect in a superconducting ring, Phys. Rev. B 2, 109 (1970).

[34] C. W. J. Beenakker, in Transport Phenomena in Mesoscopic Systems: Proceedings of the 14th Taniguchi Symposium, Shima, 1991, edited by H. Fukuyama and T. Ando (Springer, Berlin, 1992), p. 235.

[35] L. N. Bulaevskii, A. I. Buzdin, and S. V. Panjukov, The oscillation dependence of the critical current on the exchange field of ferromagnetic metals (F) in Josephson junction S-F-S, Solid State Commun. 44, 539 (1982).

[36] N. M. Chtchelkatchev, W. Belzig, Y. V. Nazarov, and C. Bruder, $\pi-0$ transition in superconductor-ferromagnet-superconductor junctions, J. Exp. Theor. Phys. Lett. 74, 323 (2001).

[37] V. V. Ryazanov, V. A. Oboznov, A. Y. Rusanov, A. V. Veretennikov, A. A. Golubov, and J. Aarts, Coupling of Two Superconductors Through a Ferromagnet: Evidence for a $\pi$ Junction, Phys. Rev. Lett. 86, 2427 (2001).

[38] R. S. Keizer, S. T. B. Goennenwein, T. M. Klapwijk, G. Miao, G. Xiao, and A. Gupta, A spin triplet supercurrent through the half-metallic ferromagnet $\mathrm{CrO}_{2}$, Nature (London) 439, 825 (2006).

[39] M. Eschrig and T. Löfwander, Triplet supercurrents in clean and disordered half-metallic ferromagnets, Nat. Phys. 4, 138 (2008).

[40] I. V. Bobkova and A. M. Bobkov, Triplet contribution to the Josephson current in the nonequilibrium superconductor/ferromagnet/superconductor junction, Phys. Rev. B 82, 024515 (2010).

[41] J. W. A. Robinson, J. D. S. Witt, and M. G. Blamire, Controlled injection of spin-triplet supercurrents into a strong ferromagnet, Science 329, 59 (2010).

[42] M. S. Anwar, F. Czeschka, M. Hesselberth, M. Porcu, and J. Aarts, Long-range supercurrents through half-metallic ferromagnetic $\mathrm{CrO}_{2}$, Phys. Rev. B 82, 100501(R) (2010).

[43] T. S. Khaire, M. A. Khasawneh, W. P. Pratt, and N. O. Birge, Observation of Spin-Triplet Superconductivity in Co-Based Josephson Junctions, Phys. Rev. Lett. 104, 137002 (2010).

[44] M. S. Anwar, M. Veldhorst, A. Brinkman, and J. Aarts, Long range supercurrents in ferromagnetic $\mathrm{CrO}_{2}$ using a multilayer contact structure, Appl. Phys. Lett. 100, 052602 (2012).

[45] A. A. Golubov, M. Y. Kupriyanov, and E. Il'ichev, The currentphase relation in Josephson junctions, Rev. Mod. Phys. 76, 411 (2004).

[46] V. Braude and Y. V. Nazarov, Fully Developed Triplet Proximity Effect, Phys. Rev. Lett. 98, 077003 (2007).

[47] L. Klam, A. Epp, W. Chen, M. Sigrist, and D. Manske, Josephson effect and triplet-singlet ratio of noncentrosymmetric superconductors, Phys. Rev. B 89, 174505 (2014). 
[48] R. Delagrange, R. Weil, A. Kasumov, M. Ferrier, H. Bouchiat, and R. Deblock, $0-\pi$ quantum transition in a carbon nanotube Josephson junction: Universal phase dependence and orbital degeneracy, Physica B 536, 211 (2018).

[49] J. J. A. Baselmans, A. F. Morpurgo, B. J. van Wees, and T. M. Klapwijk, Tunable supercurrent in superconductor/normal metal/superconductor Josephson junctions, Superlatt. Microstruct. 25, 973 (1999).

[50] T. Kontos, M. Aprili, J. Lesueur, F. Genêt, B. Stephanidis, and R. Boursier, Josephson Junction through a Thin Ferromagnetic Layer: Negative Coupling, Phys. Rev. Lett. 89, 137007 (2002).

[51] M. Houzet and A. I. Buzdin, Long range triplet Josephson effect through a ferromagnetic trilayer, Phys. Rev. B 76, 060504(R) (2007).

[52] L. B. Ioffe, V. B. Geshkenbein, M. V. Feigel'man, A. L. Fauchère, and G. Blatter, Environmentally decoupled $s d s$-wave Josephson junctions for quantum computing, Nature (London) 398, 679 (1999).

[53] G. Blatter, V. B. Geshkenbein, and L. B. Ioffe, Design aspects of superconducting-phase quantum bits, Phys. Rev. B 63, 174511 (2001).

[54] T. Yamashita, K. Tanikawa, S. Takahashi, and S. Maekawa, Superconducting $\pi$ Qubit with a Ferromagnetic Josephson Junction, Phys. Rev. Lett. 95, 097001 (2005).

[55] B. M. Niedzielski, E. C. Gingrich, R. Loloee, W. P. Pratt, and N. O. Birge, S/F/S Josephson junctions with single-domain ferromagnets for memory applications, Supercond. Sci. Technol. 28, 085012 (2015).

[56] E. C. Gingrich, B. M. Niedzielski, J. A. Glick, Y. Wang, D. L. Miller, R. Loloee, W. P. Pratt, Jr., and N. O. Birge, Controllable $0-\pi$ Josephson junctions containing a ferromagnetic spin valve, Nat. Phys. 12, 564 (2016).

[57] S. Diesch, P. Machon, M. Wolz, C. Sürgers, D. Beckmann, W. Belzig, and E. Scheer, Creation of equal-spin triplet superconductivity at the $\mathrm{Al} / \mathrm{EuS}$ interface, Nat. Commun. 9, 5248 (2018).

[58] A. Kadigrobov, R. I. Shekhter, and M. Jonson, Quantum spin fluctuations as a source of long-range proximity effects in diffusive ferromagnet-super conductor structures, Europhys. Lett. 54, 394 (2001).

[59] F. S. Bergeret, A. F. Volkov, and K. B. Efetov, Long-Range Proximity Effects in Superconductor-Ferromagnet Structures, Phys. Rev. Lett. 86, 4096 (2001).

[60] F. S. Bergeret, A. F. Volkov, and K. B. Efetov, Josephson current in superconductor-ferromagnet structures with a nonhomogeneous magnetization, Phys. Rev. B 64, 134506 (2001).

[61] A. F. Volkov, F. S. Bergeret, and K. B. Efetov, Odd triplet Superconductivity in Superconductor-Ferromagnet Multilayered Structures, Phys. Rev. Lett. 90, 117006 (2003).
[62] F. S. Bergeret, A. F. Volkov, and K. B. Efetov, Odd triplet superconductivity and related phenomena in superconductorferromagnet structures, Rev. Mod. Phys. 77, 1321 (2005).

[63] T. E. Baker, A. Richie-Halford, and A. Bill, Long range triplet Josephson current and $0-\pi$ transitions in tunable domain walls, New J. Phys. 16, 093048 (2014).

[64] M. Eschrig, Spin-polarized supercurrents for spintronics, Phys. Today 64 (1), 43 (2011)

[65] J. Linder and J. W. A. Robinson, Superconducting spintronics, Nat. Phys. 11, 307 (2015).

[66] M. Eschrig, Spin-polarized supercurrents for spintronics: A review of current progress, Rep. Prog. Phys. 78, 104501 (2015).

[67] A. I. Larkin and Yu. N. Ovchinnikov, Quasiclassical method in the theory of superconductivity, Sov. Phys._JETP 28, 1200 (1969).

[68] A. L. Shelankov, On the derivation of quasiclassical equations for superconductors, J. Low Temp. Phys. 60, 29 (1985).

[69] J. Rammer and H. Smith, Quantum field-theoretical methods in transport theory of metals, Rev. Mod. Phys. 58, 323 (1986).

[70] C. J. Lambert and R. Raimondi, Phase-coherent transport in hybrid superconducting nanostructures, J. Phys.: Condens. Matter 10, 901 (1998).

[71] W. Belzig, F. K. Wilhelm, C. Bruder, G. Schön, and A. D. Zaikin, Quasiclassical Green's function approach to mesoscopic superconductivity, Superlatt. Microstruct. 25, 1251 (1999).

[72] Y. V. Nazarov, Circuit Theory of Andreev Conductance, Phys. Rev. Lett. 73, 1420 (1994).

[73] Y. V. Nazarov, Novel circuit theory of Andreev reflection, Superlatt. Microstruct. 25, 1221 (1999).

[74] R. C. Dynes, J. P. Garno, G. B. Hertel, and T. P. Orlando, Tunneling Study of Superconductivity near the Metal-Insulator Transition, Phys. Rev. Lett. 53, 2437 (1984).

[75] J. P. Morten, D. Huertas-Hernando, W. Belzig, and A. Brataas, Full counting statistics of crossed Andreev reflection, Phys. Rev. B 78, 224515 (2008).

[76] M. Alidoust, J. Linder, G. Rashedi, T. Yokoyama, and A. Sudbø, Spin-polarized Josephson current in superconductor/ferromagnet/superconductor junctions with inhomogeneous magnetization, Phys. Rev. B 81, 014512 (2010).

[77] K. Halterman and M. Alidoust, Josephson currents and spintransfer torques in ballistic SFSFS nanojunctions, Supercond. Sci. Technol. 29, 055007 (2016).

[78] J.-C. Tung and G.-Y. Guo, High spin polarization of the anomalous Hall current in Co-based Heusler compounds, New J. Phys. 15, 033014 (2013).

[79] L. Bainsla, K. G. Suresh, A. K. Nigam, M. Manivel Raja, B. S. D. C. S. Varaprasad, Y. K. Takahashi, and K. Hono, High spin polarization in $\mathrm{CoFeMnGe}$ equiatomic quaternary Heusler alloy, J. Appl. Phys. 116, 203902 (2014). 